\title{
A Case of Gardner's Syndrome Associated with Desmoid Tumor
}

\author{
Jimin Chung, M.D., Sun Namkoong, M.D., Kyoung Eun Jung, M.D., Jong Wook Park, M.D., \\ Byung Cheol Park, M.D., Yong Woo Cinn, M.D., Myung Hwa Kim, M.D. \\ Department of Dermatology, College of Medicine, Dankook University, Cheonan, Korea
}

Desmoid tumors are uncommon benign neoplasm of the fibroblasts. They occur rarely in the general population, but they are comparatively common in patients with familial polyposis coli with or without other elements of Gardner's syndrome. Herein, we report a 16-year-old woman with Gardner's syndrome complicated by desmoid tumors on the right subscapular area. (Ann Dermatol 22(4) 418 421, 2010)

\section{-Keywords-}

Desmoid tumor, Familial polyposis coli, Gardner's syndrome

\section{INTRODUCTION}

Desmoid tumors are benign tumors exhibiting fibroblastic proliferation that arise from fascial or musculoaponeurotic structures. They are rare in the general population, accounting for $0.03 \%$ of all neoplasm, although they occur more commonly in familial adenomatous polyposis (FAP) patients, exhibiting an incidence of $3.5 \sim 32 \%$, and the incidence is $29 \%$ in the original Gardner kindred ${ }^{1,2}$.

Gardner's syndrome is an autosomal dominant inherited condition, characterized by polyposis coli, osteoma and various soft tissue tumors, including desmoid tumors. We herein report a woman with Gardner's syndrome associated with desmoids tumor.

Received April 29, 2009, Revised August 31, 2009, Accepted for publication September 9, 2009

Corresponding author: Myung Hwa Kim, M.D., Department of Dermatology, Dankook University Hospital, 359 Manghyangno, Dongnamgu, Cheonan 330-715, Korea. Tel: 82-41-550-6485, Fax: 82-41-5527541, E-mail: ivymyung@hanmail.net

\section{CASE REPORT}

In January 2008, a 16-year-old girl visited our clinic complaining of a palpable mass on right subscapular area developed since birth. She was under evaluation with incomplete development of teeth at the oral surgery department, and was referred to our department to evaluate the skin tumor. The patient had been in her usual state of health, and had no remarkable past medical history. None of her family members had known health problems or any hereditary diseases.

Skin examination revealed a non-tender, hard, skin colored, elevated and well-confined mass on the right subscapular area, measuring $7 \times 4 \mathrm{~cm}$ (Fig. 1). All laboratory examinations on blood and urine were within the normal limits. Computer tomography of the oropharynx showed polyostotic fibrous dysplasia involving facial, skull base and cranial bones (Fig. 2). Gastrofibroscopy and colonoscopy revealed numerous small polyps in the stomach and colon (Fig. 3). Polyps in colon were tubular adenomas histopathologically.

Skin biopsy was performed in the middle of the subscapular mass, which showed dense collagen bundles in dermis, and among thickened collagen bundles were ongoing fibroblasts normal differentiation (Fig. 4). Cytogenetic studies showed nonsense mutation of the APC gene on the 4,585th base sequence of exon 15 . Studies on her family members were not performed.

Finally, she was diagnosed with the Gardner's syndrome according to fibrous dysplasia of facial bone, polyposis of stomach and colon, subscapular desmoid tumor and mutation of APC gene. She is now under observation with periodic colonoscopy, without any progression of disease so far. 

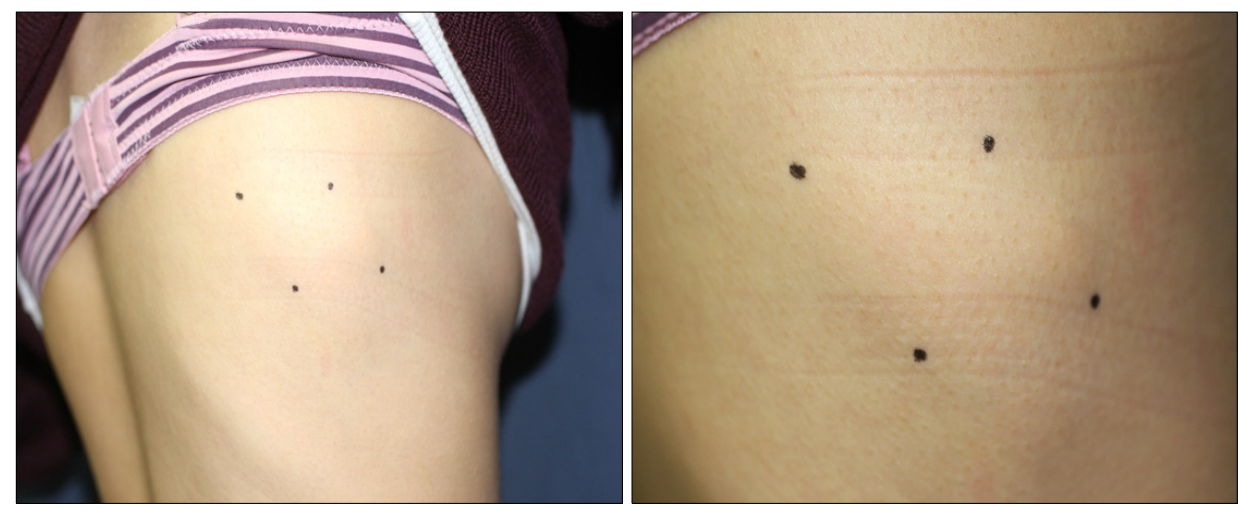

Fig. 1. Skin colored elevated tumor on right subscapular area, measuring $7 \times 4 \mathrm{~cm}$.
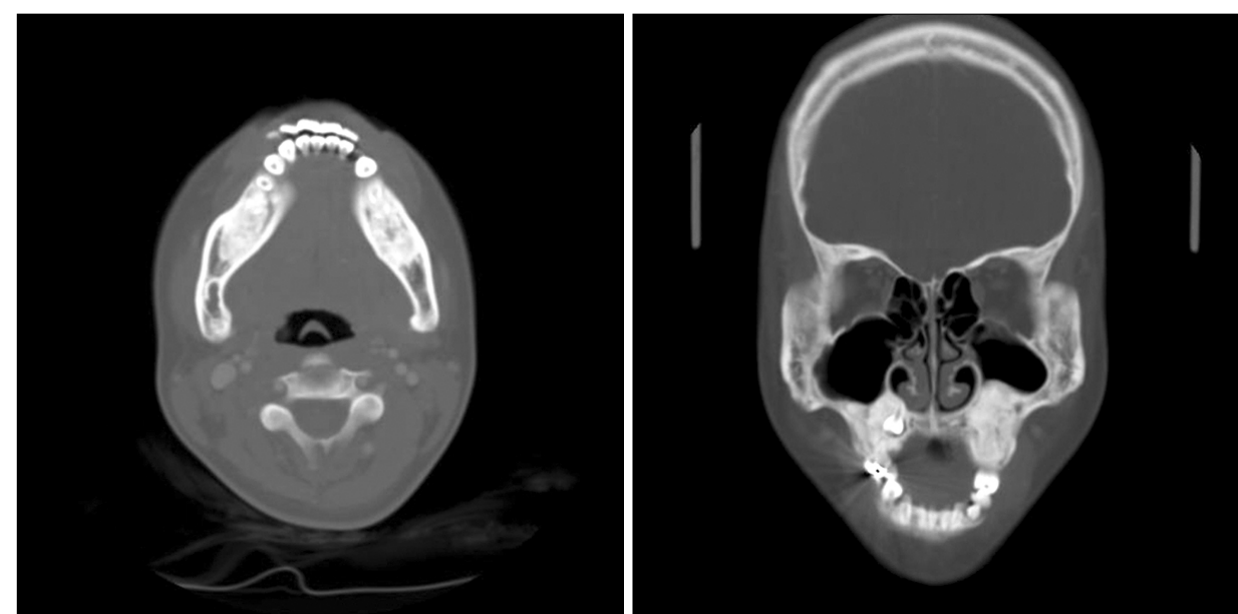

Fig. 2. Fibrous dysplasia involving facial and cranial bones.
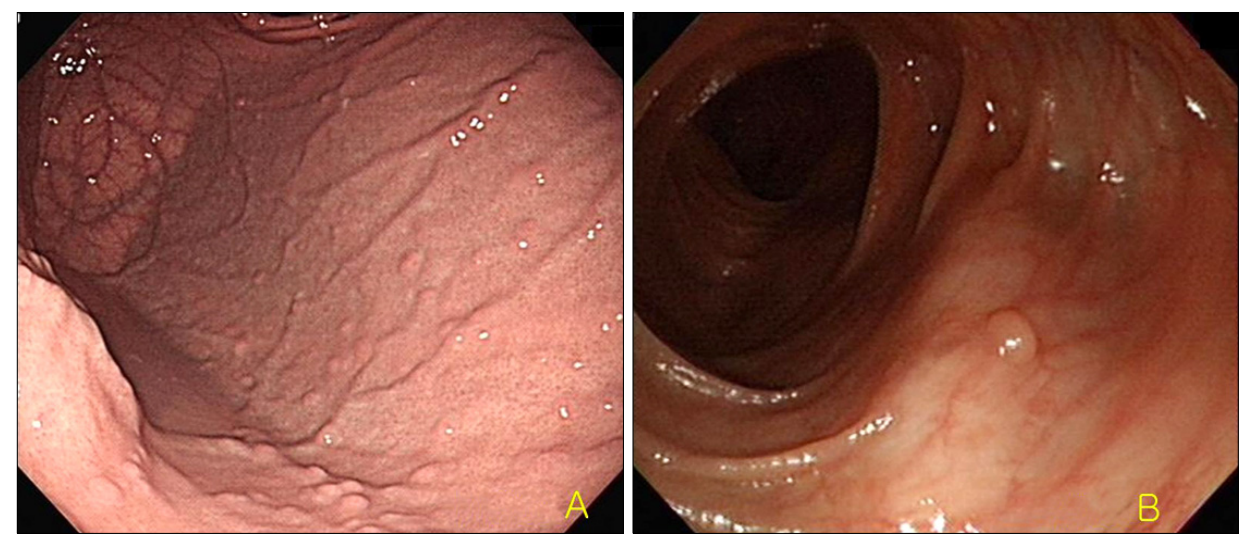

Fig. 3. Endoscopic findings. Numerous small polyps are observed in stomach (A) and colon (B).

\section{DISCUSSION}

Gardner's syndrome was first reported by Gardner and Smith in 1958. It is an autosomal dominant, non-sex linked, complex condition involving soft-tissue masses, bony tumors, and polyposis coli $\mathrm{i}^{1,3}$. It is considered to be a variant of FAP, as both entities are caused by mutations in the APC gene located at band q21 on chromosome 5, of which $20 \%$ are de novo in nature ${ }^{4}$.
Extracolonic manifestations include osteomas involving the maxilla, the mandible, long bones, cranial bones and the sinuses, epidermoid cysts, lipomas, dental anomalies such as impacted and supernumerary teeth. There are also central nerve system tumors, thyroid gland adenomas and carcinomas, adrenal gland adenomas and carcinomas, upper gastrointestinal polyps, duodenal, pancreatic, liver and biliary neoplasm, carcinoid of the small bowel, lymphoid hyperplasia of the terminal ileum and congeni- 

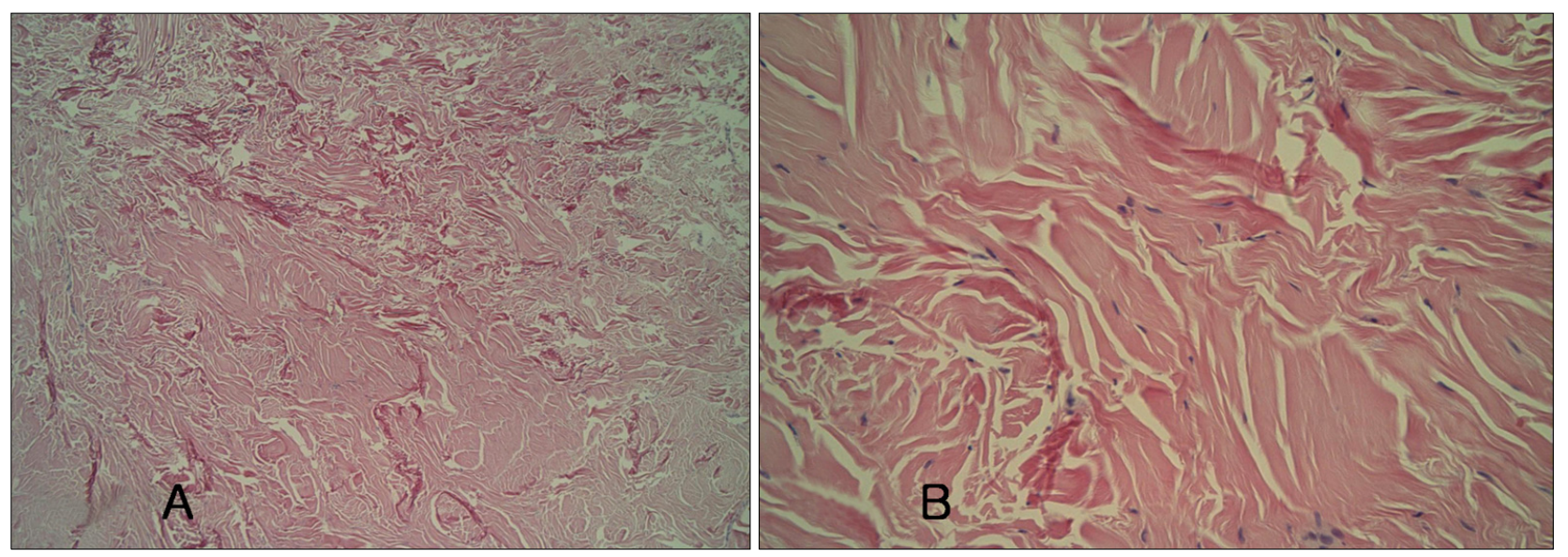

Fig. 4. Histopathology of right subscapular tumor. (A) Dense collagen bundles in dermis $(H \& E, \times 40)$. (B) Thickened collagen bundles with interspersed spindle cells and fibroblast $(\mathrm{H} \& \mathrm{E}, \times 200)$.

tal hypertrophy of the retinal pigment epithelium ${ }^{5}$, and desmoids tumors.

About $2 \%$ of desmoid tumors are FAP-associated, and patients with FAP are at 852 times the risk of developing desmoids tumors in the general population ${ }^{2}$. Although etiological factors in desmoids tumors are unclear, three factors including, initiation of trauma, influence of sex steroids and an underlying genetic defect, have been implicated in the etiology of desmoids tumors ${ }^{2}$.

Based on their location, desmoids tumors can be divided into three types, intra-abdominal, abdominal wall and extra-abdominal, in order of frequency. Symptoms depend on the location of the tumor. Many studies have shown that between $37 \%$ and $50 \%$ of desmoids arise in the abdominal region ${ }^{6}$. Intra-abdominal desmoids can remain asymptomatic for a considerable period, while relentlessly enlarging and infiltrating adjacent structures causing intestinal, vascular and urinary obstruction or neural involvement ${ }^{7}$. The most common extra-abdominal sites include the shoulder girdle, chest wall and inguinal regions. Pathologically, desmoids tumors are similar to other fibromatoses, including scar hypertrophy, mesenteric fibromatosis, Dupuytren's contracture, and low grade sarcoma. They are arising from the musculoaponeurotic soft tissues, and composed of well-differentiated and uniform collagenous fibroblasts and fibrocytes. They are locally invasive but do not metastasize $\mathrm{e}^{2,6-8}$.

Because Gardner's syndrome is characterized by colorectal cancer in early adult life secondary to extensive adenomatous polyps of the colon, patients need to undergo surgery. Total proctocolectomy with ileostomy or colectomy with ileorectal anastomosis is recommended ${ }^{5,9}$. Treatment of desmoids tumor is controversial. Although surgery is widely accepted as the first line treatment, the high recurrence rate has led some authors to suggest that surgery should be avoided, except for bypass of bowel obstructions or relief of ureteric obstructions caused by intraabdominal desmoids tumors. Pharmacological pain control with non-steroid anti-inflammatory drugs and anti-estrogen agent is the most widely documented management. Chemotherapy and radiotherapy are also alternatives considered in symptomatic patients who do not response to conventional medical therapy ${ }^{2,10}$.

As far as we know, a 55-year-old woman has been reported as Gardner syndrome associated with desmoids tumor so far who experienced FAP, osteomatous jaw and ocular lesion, numerous dental abnormalities and abdominal tumor ${ }^{11}$. Our patient had fibrous dysplasia of the facial bone, polyposis of stomach and colon, and subscapular desmoid tumor revealing mutation of APC gene. We report this 16-year-old woman with Gardner's syndrome complicated by desmoid tumors on the right subscapular area, a rare case in Korea.

\section{REFERENCES}

1. Smith WG. Multiple polyposis, Gardner's syndrome and desmoid tumors. Dis Colon Rectum 1958;1:323-332.

2. Tseng KC, Lin CW, Tzeng JE, Feng WF, Hsieh YH, Chou AL, et al. Gardner's syndrome--emphasis on desmoid tumors. Tzu Chi Med J 2006;18:57-60.

3. Gardner EJ. A genetic and clinical study of intestinal polyposis, a predisposing factor for carcinoma of the colon and rectum. Am J Hum Genet 1951;3:167-176.

4. Béroud C, Soussi T. APC gene: database of germline and somatic mutations in human tumors and cell lines. Nucleic Acids Res 1996;24:121-124.

5. Lin RC, Chiu HH, Jao YTFN, Liu YW, Huang TC, Mo LR, et al. Carcinoma of ampulla of vater and recurrent desmoid 
tumors in Gardner's syndrome. J Intern Med Taiwan 2008; 19:158-163.

6. Shields CJ, Winter DC, Kirwan WO, Redmond HP. Desmoid tumours. Eur J Surg Oncol 2001;27:701-706.

7. Rampone B, Pedrazzani C, Marrelli D, Pinto E, Roviello F. Updates on abdominal desmoid tumors. World J Gastroenterol 2007; 13:5985-5988.

8. Zissiadis A, Harlaftis N, Aletras H. Desmoid tumor in Gardner's syndrome. Am Surg 1990;56:305-307.

9. Hatzimarkou A, Filippou D, Papadopoulos V, Filippou G,
Rizos S, Skandalakis P. Desmoid tumor in Gardner's syndrome presented as acute abdomen. World J Surg Oncol 2006;4:18.

10. Belliveau P, Graham AM. Mesenteric desmoid tumor in Gardner's syndrome treated by sulindac. Dis Colon Rectum 1984;27:53-54.

11. Lee BD, Lee W, Oh SH, Min SK, Kim EC. A case report of Gardner syndrome with hereditary widespread osteomatous jaw lesions. Oral Surg Oral Med Oral Pathol Oral Radiol Endod 2009;107:e68-e72. 